\title{
Saccadic selection and crowding in visual search: stronger lateral masking leads to shorter search times
}

\author{
Jelmer P. de Vries • Ignace T. C. Hooge • \\ Marco A. Wiering $\cdot$ Frans A. J. Verstraten
}

Received: 19 July 2010 / Accepted: 24 March 2011 / Published online: 12 April 2011

(C) The Author(s) 2011. This article is published with open access at Springerlink.com

\begin{abstract}
We investigated the role of crowding in saccadic selection during visual search. To guide eye movements, often information from the visual periphery is used. Crowding is known to deteriorate the quality of peripheral information. In four search experiments, we studied the role of crowding, by accompanying individual search elements by flankers. Varying the difference between target and flankers allowed us to manipulate crowding strength throughout the stimulus. We found that eye movements are biased toward areas with little crowding for conditions where a target could be discriminated peripherally. Interestingly, for conditions in which the target could not be discriminated peripherally, this bias reversed to areas with strong crowding. This led to shorter search times for a target presented in areas with stronger crowding, compared to a target presented in areas with less crowding. These findings suggest a dual role for crowding in visual search. The presence of flankers similar to the target deteriorates the quality of the peripheral target signal but can also attract eye movements, as more potential targets are present over the area.
\end{abstract}

Keywords Visual search $\cdot$ Crowding $\cdot$ Saccadic selection $\cdot$ Potential targets

J. P. de Vries $(\varangle)$ · I. T. C. Hooge · F. A. J. Verstraten

Department of Experimental Psychology,

Helmholtz Institute, Utrecht Neuroscience and Cognition,

Utrecht University, Heidelberglaan 2,

3584 CS Utrecht, The Netherlands

e-mail: j.p.devries@uu.nl

\section{A. Wiering}

Department of Artificial Intelligence,

Faculty of Mathematics and Natural Sciences,

University of Groningen, Groningen, The Netherlands

\section{Introduction}

A visual scene often consists of multiple objects. Finding an object in such a scene can vary in difficulty. When the object we are looking for is conspicuous, it can be discriminated peripherally without making a saccade (e.g., Wertheim et al. 2006). However, the limited resolution of our visual periphery often requires us to make eye movements to locate and inspect the objects (e.g., Yarbus 1967). Even though visual information from the periphery is often too sparse to identify targets, it is generally good enough to select candidates resembling the target for further inspection (saccadic selection). We refer to these candidates as potential targets for eye movements. After selection, a saccade to the next element is made, and foveal inspection may reveal a target. This process continues until the target is found or, when no target is present, the whole display has been inspected. Throughout many different saccadic search tasks where eye movements were measured, the nature of potential targets was studied (e.g., Findlay 1997; Hooge and Erkelens 1999; Motter and Belky 1998; McSorley and Findlay 2003). It was shown for example that color and shape are potent in limiting the set of potential targets (Luria and Strauss 1975). This results in more efficient search, as fewer elements have to be inspected. A large body of research has been devoted to guidance in search, relating stimulus properties to search efficiency. We now know that search for a target defined by a combination of two features is inefficient, as these features are not automatically combined in the periphery (Treisman and Gelade 1980). Also, decreasing target-distractor dissimilarity slows search (Duncan and Humphreys 1989). Emphasizing the ability to guide search, Bacon and Egeth (1997) showed observers informed on the frequency of different distractor types could weigh selection toward particular target 
features. A comprehensive overview of many stimulus properties influencing search efficiency can be found in Guided Search 4.0 by Wolfe (2007).

Many models of visual search assume that features of elements in the periphery are available (e.g., Avraham et al. 2008; Wolfe 2007). However, elements near each other in the periphery impair the ability to detect properties of those elements, a phenomenon known as crowding (e.g., Bouma 1970; Toet and Levi 1992). In saccadic search, the presence of flankers around search elements deteriorates search performance (Vlaskamp and Hooge 2006). These authors suggest that crowding rather than decreasing retinal resolution is the bottleneck in visual search. However, from this study, it is not clear whether crowding affected saccadic selection or peripheral target discrimination. Recent studies on eye movements in complex search tasks find saliency to be an important factor in saccadic selection, regardless of the properties of the target. Neider and Zelinsky (2006) varied target-background similarity and found a bias in saccadic selection. Salient patterns segmented from the background were fixated more frequently than target-similar unsegmented regions of the background. Furthermore, in a study using rhesus monkeys, Shen and Pare (2006) found an inverse relation between the proportion of target-colored elements in the stimulus and the proportion of initial eye movements landing on them. Hence, initial eye movements were biased to elements of the color least present in the stimulus, those most likely to be salient. These findings are in contrast to saccadic selection being biased toward elements sharing features of the target, which has been found in eye movement studies with elements placed on a blank background. However, the inverse relation found in Shen and Pare (2006) concerns the initial eye movement, which is more likely to be captured by a salient element than subsequent eye movements (Findlay and Brown 2006). Also, in daily life, the distinction between objects and background is often not as clear as in Neider and Zelinsky (2006), and scenes can be cluttered with elements similar and dissimilar to the target. The target might be found among either of these. How is the search for the target executed in such complex scenes? In the present study, we investigate how different crowding conditions affect eye movement strategies. The crowding conditions were achieved by manipulating both target and flankers.

Before hypothesizing about the effect of crowding on saccadic selection, it is important to know the properties of crowding. Crowding has extensively been studied in many psychophysical experiments. Its main properties can be summarized in three empirical laws. Most important to us is that flankers more similar to the target increase crowding (Nazir 1992; Kooi et al. 1994). Furthermore, crowding also increases when flankers are closer to the peripheral target (e.g., Toet and Levi 1992). Finally, crowding is stronger when a target, together with flankers, is located further in the visual periphery (see e.g., Bouma 1970; Butler and Currie 1986; Polat and Sagi 1993; Huckauf et al. 1999).

To generalize the effects of crowding from peripheral identification tasks to saccadic search is potentially problematic because both peripheral target discrimination and saccadic selection may be affected by crowding. Observers may adopt different eye movement strategies depending on the quality of peripheral visual information. Imagine a stimulus consisting of one target and many search elements, some of the search elements resemble the target and some do not. Throughout this stimulus, search elements are non-uniformly distributed over the display, resulting in a display with areas over which crowding varies. As stated above, the target can be found by: (1) peripheral target discrimination or (2) saccadic selection followed by foveal discrimination. We start by focusing on peripheral target discrimination. How is a target found in an area containing many elements not resembling the target? In such an area, a target can often be discriminated peripherally (target fixation is not required for target discrimination), because here peripheral discrimination does not suffer from crowding. In contrast, in an area where different distractors resemble the target, peripheral target discrimination is often not possible and the efficiency of search depends on saccadic selection. Even though crowding deteriorates the quality of peripheral information in this situation, crowding is not taken into account into current models of visual search. Furthermore, much of the guidance literature investigates search efficiency, rather than saccadic selection. Therefore, it cannot show what attracts eye movements, while eye movements can reveal search processes that are not clear from manual reaction times analysis (Zelinsky 1996). Many theories on crowding exist (for a review see Levi 2008) making it difficult to predict what eye movement strategy observers may adopt. The important question is: does the oculomotor system prefer areas containing many potential targets and strong crowding or does it prefer areas containing less potential targets and less crowding, as might be expected from recent findings in complex search tasks (Neider and Zelinsky 2006; Shen and Pare 2006; Torralba et al. 2006)?

To investigate this question, we constructed a stimulus containing search elements, each accompanied by flankers, which had no predictive power over the location of the target. By manipulating the properties of these flankers, we influenced the strength of crowding, as flankers more similar to the target cause stronger crowding. In this way, we varied the strength of crowding for different areas in the display.

\section{Experiment 1A}

Any strategy employed to find a target is likely to depend on whether a target can or cannot be discriminated periph- 
erally; therefore, we varied the properties of the target over two conditions.

In the visual periphery, sensitivity for high spatial frequencies decreases. Crowding further deteriorates the ability to discriminate elements in the periphery. Therefore, high spatial frequency elements are more likely to become indiscriminable as a result of crowding. We used spatial frequency to vary the discriminability of the target. In the low spatial frequency $(l s f)$ condition, the target is an $l s f$ Gabor patch $\left(2.6\right.$ cycles $\left./{ }^{\circ}\right)$ and can likely be discriminated despite crowding. In the high spatial frequency ( $h s f)$ condition, the target is an $h s f$ Gabor patch $\left(5.2\right.$ cycles $\left./^{\circ}\right)$ and is more likely to become indiscriminable due to crowding.

To investigate the influence of crowding, throughout both conditions, in different trials, the target can be found either among flankers of the same spatial frequency or among flankers of a different spatial frequency. In the $l s f$ condition, we expect to find a preference for locations where the target is accompanied by flankers of a different spatial frequency, as these do not require fixation in order to be discriminated. However, the most interesting case is the $h s f$ condition. The oculomotor system might again prefer areas with little crowding. However, locations with strong crowding might also be preferred, as they reveal many potential targets.

Methods

\section{Observers}

Each condition included six observers, three of which participated in both conditions. All observers had normal or corrected to normal vision and were of ages 25-42. Two observers, $I H$ and $J V$, are authors of this paper; other observers were naive as to the goal of the experiment. Observers either worked or studied at Utrecht University and participated on a voluntary basis.

\section{Apparatus and stimuli}

All stimuli consisted of 12 search elements on an invisible 4 by 3 hexagonal grid on a gray background $\left(6.0 \mathrm{~cd} / \mathrm{m}^{2}\right)$. These search elements, Gabors, consisted of one target and 11 distractors. To distinguish target from distractors, the sinusoidal carrier was rotated and modulated to change the orientation and spatial frequency, respectively. Elements were placed at a spacing of $8.1^{\circ}$ (center-to-center) and were of either high spatial frequency $\left(5.2\right.$ cycles $/{ }^{\circ}, 12.5 \mathrm{~cd} / \mathrm{m}^{2}$, $85 \%$ contrast $)$ or low spatial frequency $\left(2.6\right.$ cycles $/{ }^{\circ}$, $13.0 \mathrm{~cd} / \mathrm{m}^{2}, 80 \%$ contrast). The Gabors had a diameter of $0.92^{\circ}$ on screen; however, perceived radius was slightly smaller at approximately $0.8^{\circ}$ (see Fredericksen et al. 1997). The target had a vertical orientation, while other

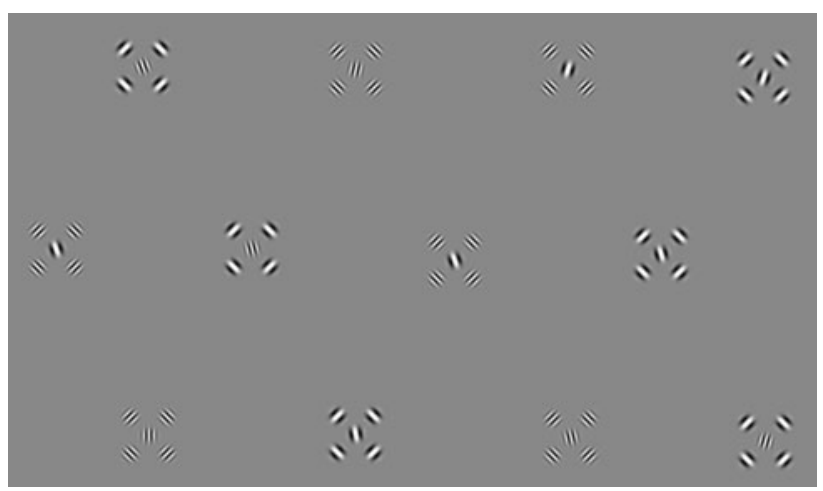

Fig. 1 An example of the stimulus used in Experiment 1A from the $h s f$ condition. The target can be found in the lower left corner of the display. Contrast is slightly enhanced for illustrative purposes

elements were rotated away from the vertical orientation (randomly chosen from a range of $10^{\circ}-20^{\circ}$ ), either clockwise or counterclockwise.

Target and distractors were, each individually, surrounded by four flankers, creating a display containing 12 clusters. A cluster subtended $2.3^{\circ}$ by $2.3^{\circ}$. Flankers were Gabors of either high spatial frequency ( $h s f$ ) or low spatial frequency $(l s f)$, resulting in four types of clusters (clusters composed of an $l s f$ center with $l s f$ flankers, an $l s f$ center with $h s f$ flankers, an $h s f$ center with $l s f$ flankers, and an $h s f$ center with $h s f$ flankers). For each cluster, they were rotated either $45^{\circ}$ clockwise (upper left and lower right flankers) or $45^{\circ}$ counterclockwise (upper right and lower left flankers). An example of the stimulus can be found in Fig. 1. The stimuli were generated using Matlab on an Apple G4 and displayed on a Lacie 22" CRT monitor at a resolution of 1,600 by 1,200 pixels with a refresh rate of $75 \mathrm{~Hz}$.

\section{Procedure}

Depending on the two conditions, observers were instructed to search for an $h s f$ or an $l s f$ vertical Gabor. Next, observers were set up with the Eyelink and instructed on the proceedings of a trial. Before each trial, a small fixation cross was displayed and observers started a trial by pressing the space bar. Observers indicated having located the target pressing the "zero key" on the numerical keypad. Each condition lasted approximately $15 \mathrm{~min}$ and a break of at least $5 \mathrm{~min}$ between conditions was included.

\section{Eye movement analysis}

Eye movements were recorded using an SMI Eyelink II system at a frequency of $500 \mathrm{~Hz}$, while the observer's head was placed in a chinrest at a distance of $64 \mathrm{~cm}$ to the screen. Images were viewed binocularly, but eye movements were recorded from the left eye only. Eye movement data were 
Fig. 2 Median saccade amplitudes set out against each cluster type. a The different types of clusters in the low spatial frequency target condition. b Saccade amplitudes toward the different types of clusters in the high spatial frequency target condition. Letters represent cluster compositions. An $L$ indicates low spatial frequency Gabors, and an $H$ indicates high spatial frequency Gabors
(A)

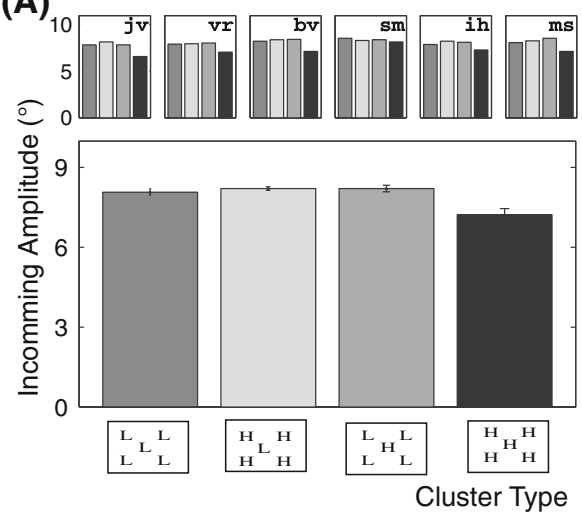

(B)

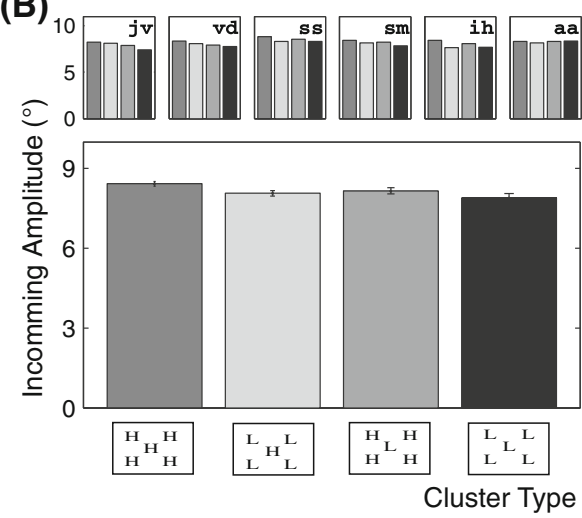

collected for off-line analysis. Saccades were detected at a velocity of $20 \%$ s, after which start and endpoint were found by searching back and forth until the velocity was two standard deviations higher than the velocity during fixations (as in Van der Steen and Bruno 1995). Saccades with amplitudes under $1.5^{\circ}$ were removed from the analysis. If a small saccade was removed, fixations before and after this saccade were added together. Finally, fixation durations shorter than $50 \mathrm{~ms}$ were discarded from further analysis.

\section{Results}

\section{Saccade amplitudes}

The goal of the experiment was to evaluate the outcome of the selection process. To draw any conclusions from the results of our experiments on the selection process, it is important that selection is based on a similar choice for each eye movement. A possible confounding factor for these displays was that eye movements targeting one type of cluster originated from far away, while another type of cluster was only targeted when it was a direct neighbor. The distance between clusters and the properties of the Gabors were chosen such that observers could only evaluate one cluster per fixation and saccade to neighboring clusters.

Saccade amplitudes are generally acknowledged to be a good indication of the number of stimulus elements that can be evaluated in a single fixation (e.g. Jacobs 1986). In Fig. 2, we plot the median saccade amplitudes to verify that they are in accordance with the intention of saccades targeting neighboring clusters. Here, we see that the median saccade amplitudes are similar for all clusters, indicating that different types of clusters are not selected from different distances.

\section{Search time}

In order to evaluate the influence of flankers on selection for each condition, we divided the trials into two subsets of which we averaged search times individually. The subsets are based on the type of flanker, either $h s f$ or $l s f$, surrounding the target. Note that only two of the four cluster types, those with the correct spatial frequency, could hold the target. This allows us to distinguish search times for a target surrounded by similar flankers from a target surrounded by dissimilar flankers. A bias in eye movements toward one type of cluster should result in shorter search times when the target is found in such a cluster, as it then has a higher chance of being fixated. In Fig. 3a, search times are plotted for the $l s f$ condition. In this condition, finding a target among similar flankers requires longer search times than finding a target among dissimilar flankers $(t(5)=4.389$; $P<0.01)$. This is contrasted by the results for the $h s f$ condition (Fig. 3b). Here, we see longer search times when the flankers of the target are dissimilar in comparison with shorter search times for a target placed among similar flankers $(t(5)=3.121 ; P<0.05)$.

\section{Selection of clusters}

If the difference in search times is indeed due to a bias in selection based on the type of flankers, a difference in the number of fixations per cluster type should be at the basis of this variation in search times. That is, we reason a target to be found quicker in a cluster type, if this cluster is more likely to be fixated. Therefore, we analyzed which clusters were fixated throughout a search. In Fig. 4, the proportion of the total number of fixations is plotted for each of the four types of clusters. When a target is not found in the clusters that attract eye movements, naturally the rest of the clusters will also be scanned. As our interest lies with the initial preference and not the systematic scanning of the entire image, this analysis was limited to the endpoints of the first three saccades.

In the $l s f$ condition, clusters where an $l s f$ Gabor is accompanied by dissimilar, $h s f$, flankers are fixated more frequently than clusters with similar, $l s f$, flankers. However, 
(A)

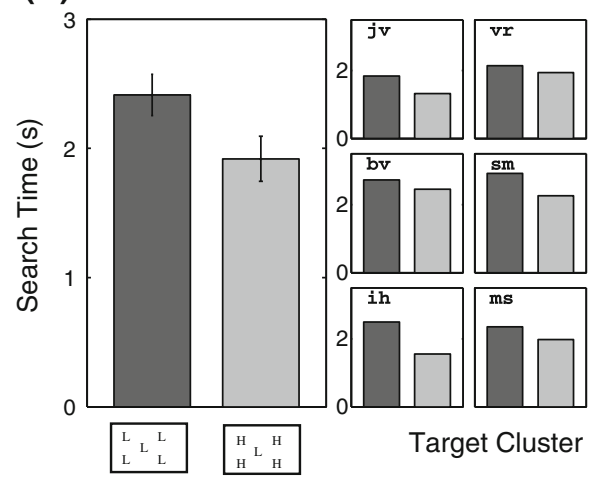

Fig. 3 Search times for the first experiment. a Average search times for low spatial frequency target. Trials are divided into two subsets, dark gray bars indicate search times when the target is surrounded by similar, low spatial, flankers. Light gray bars indicate search times when target is surrounded by dissimilar, high spatial frequency, flankers. b The search times for the high spatial frequency target. Dark bars
(B)

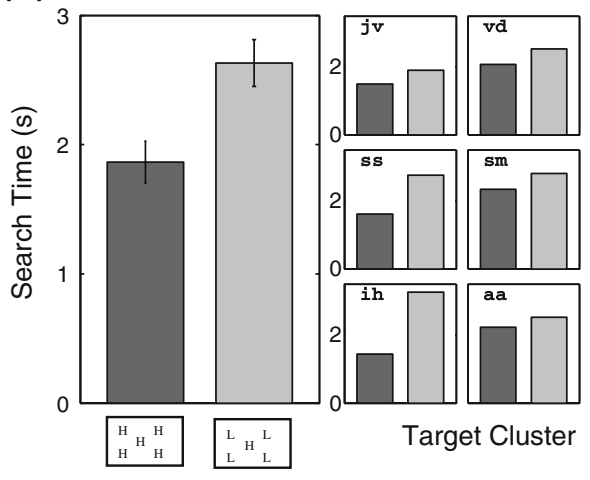

again indicate search times when the target is surrounded by similar, in this case high spatial frequency, flankers. Light gray bars indicate search times when the target is surrounded by dissimilar, low spatial frequency, flankers. Cluster composition is indicated using letters. The letter $L$ represents low spatial frequency Gabors, the $H$ high spatial frequency Gabors. Error bars represent standard errors of the mean
Fig. 4 Number of fixations set out for the four cluster types. a The results for the low spatial frequency target condition. b The results for the high spatial frequency target condition. Letters represent cluster compositions. An $L$ indicates low spatial frequency Gabors, and an $H$ indicates high spatial frequency Gabors. Error bars represent standard deviations
(A)
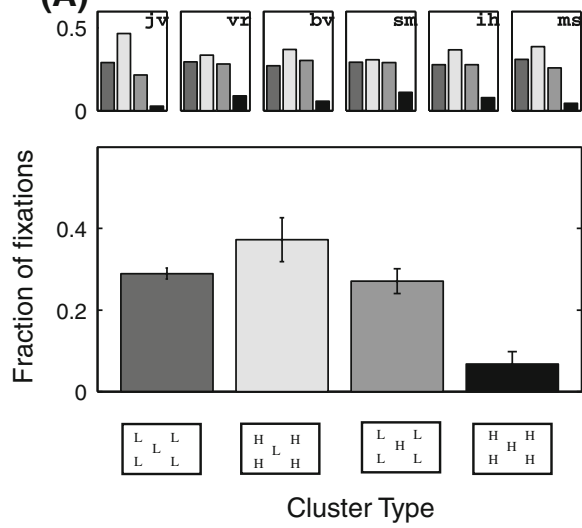

(B)
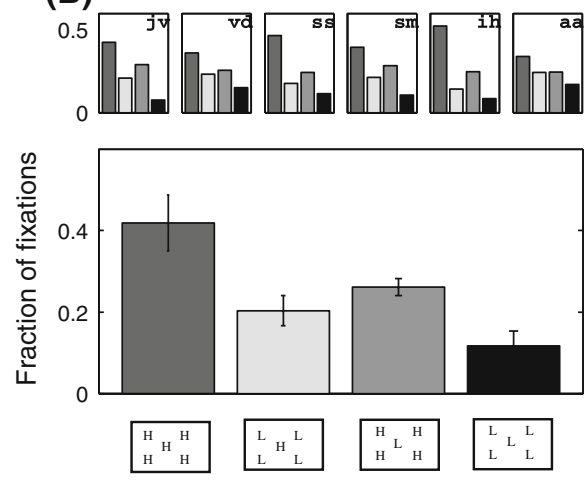

Cluster Type during the search for an $h s f$ target, clusters with similar, $h s f$, flankers are fixated more frequently than clusters with dissimilar, lsf, flankers. This held even when a dissimilar, lsf, Gabor is located at the center of the cluster.

\section{Discussion: Experiment 1A}

Comparing the two conditions, search times for the $l s f$ condition are shorter than those for the $h s f$ condition. However, as we are interested in saccadic selection, we focus on where in the display the target is found quickest, rather than in what condition. In the $l s f$ condition, we see that search times are shorter when an $l s f$ target is flanked by $h s f$ Gabors, than when it is flanked by $l s f$ Gabors. This can be explained as flankers less similar to the target form weaker masks (Nazir 1992; Kooi et al. 1994). However, ordinary masking cannot explain why a target is found fastest when surrounded by similar flankers in the hsf target condition.
To explain this counterintuitive result, we need to move beyond the idea of just masking of the target. In this case, it seems that surroundings can no longer be distinguished from the target, and saccadic targeting seems to be attracted by the properties of the central and flanking Gabors combined. Locations where more of the target property is present over a greater area are now more likely to be selected by saccadic targeting. These results suggest that when a target is peripherally indiscriminable, areas with more potential targets and stronger crowding are preferred over those with less potential targets and less crowding. This is in contrast to saccadic selection being guided by saliency, as clusters with irregularities (those with a combination of $h s f$ and $l s f$ Gabors) are selected less frequently than the homogenous clusters with elements similar to the target. However, one remaining issue is that this result can also be explained in terms of the type of flanker, rather than the similarity it has to its center. We will address this issue in Experiment 2. 
Fig. 5 Conspicuity angles for the $l s f$ and $h s f$ target in $\mathbf{a}$ and $\mathbf{b}$, respectively. $S$ denotes the target is surrounded by similar flankers, $D$ by dissimilar flankers, and $N$ by no flankers. Error bars of the average conspicuity angles represent standard error of the mean, and error bars of individual observers represent standard deviations

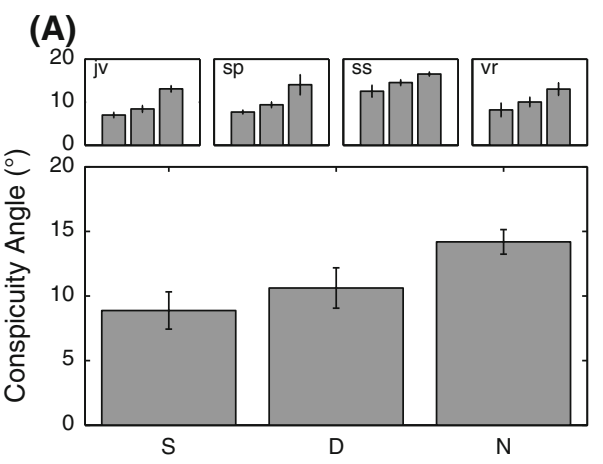

(B)

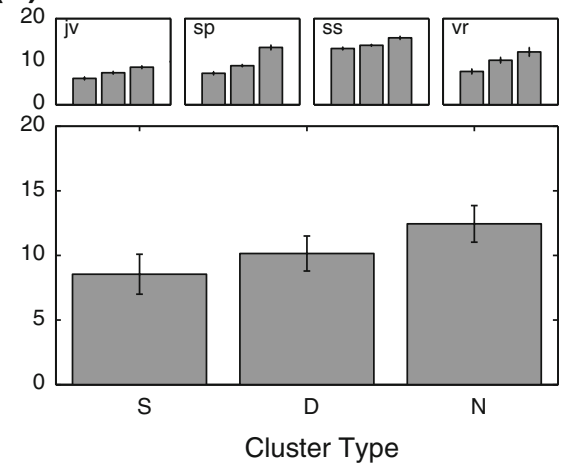

\section{Experiment 1B}

On the basis of literature, we assumed that in our experiment flankers more similar to the target always form the strongest mask. Considering the many other factors, apart from spatial frequency, that can be of influence in masking, such as luminance and contrast, we performed a small control experiment in order to check the validity of our assumption for the stimuli used. The measurements were taken using a method based on the conspicuity meter, as introduced by Wertheim et al. (2006). This procedure is subjective, as observers are asked to indicate at what eccentricity they can no longer recognize the target. However, as we are interested in comparing between different cluster compositions, rather than finding absolute conspicuity angles, this method serves our goal best.

\section{Methods}

\section{Observers}

Four observers from the same pool as the previous experiment participated in the task.

\section{Stimulus}

The stimulus consists of a fixation cross with a peripherally placed cluster (either to the left or the right of the fixation cross). The cluster is initially placed at an eccentricity of $16.2^{\circ}$ near the border of the screen. Located at the center of the cluster is a vertical target Gabor as used in Experiment $1 \mathrm{~A}$. Either $l s f$ or $h s f$ flankers surround this target. A condition where no flankers were present was also added to obtain a complete picture of the influence of the flanking Gabors on peripheral discrimination.

\section{Procedure}

For each type of cluster, observers performed this task 6 times, 3 times on the left side of the fixation cross and 3 times on the right side of the fixation cross. They were asked to place the Gabor at the largest eccentricity that still allows them to distinguish it as the vertical target, while fixating the central fixation cross. The cluster can be moved toward the center as well as away from fixation by using the arrow keys.

\section{Results}

Figure 5 shows the eccentricities of the conspicuity measurements for the four observers. In Fig. 5a, b, the results are plotted for the lsf and the $h s f$ target condition, respectively. The same patterns were found not only for all observers within a condition but also for both conditions. Conspicuity angles are the smallest when flankers of the same spatial frequency surround the target. Conspicuity angles are higher when dissimilar flankers surround target and are highest when no flankers are presented around the target.

\section{Discussion: Experiment 1B}

This control experiment shows that the presence of flankers decreases the eccentricity at which observers can still recognize the target. In line with earlier studies, we see that this eccentricity is smaller for flankers that are more similar to the target.

As the decreasing resolution of peripheral vision allows for relatively better discrimination of lower spatial frequencies, it seems counterintuitive that conspicuity angles for the $h s f$ target and the $l s f$ target are close to equal. However, this is explained by the difference in the task in the control experiment and what is likely to happen in saccadic selection. In the control experiment, the task is to distinguish the vertical orientation of the central Gabor. As discrimination thresholds are higher for lower spatial frequencies (Burr and Wijesundra 1991), the discrimination of the orientation of an $l s f$ Gabor is less accurate at any eccentricity. In the results of Experiment 1A, we evaluate selection by looking at contrast between spatial frequencies of target and flankers, 
(A)

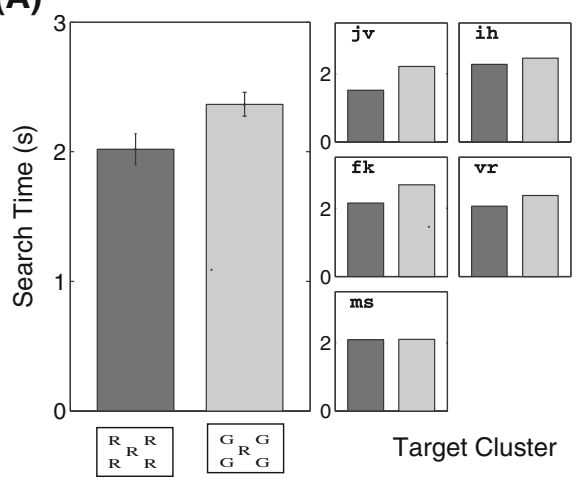

Fig. 6 Search times for the search task with as target a colored Gabor. a Results for the red target Gabor condition, $\mathbf{b}$ for the green target Gabor condition. Dark gray bars indicate times from the subset in which the target is flanked by the same color Gabors (similar flankers).

not by the distinction of the orientation of the central Gabor. However, in this experiment, we purely intended to show the difference in masking strength, rather than to provide an absolute conspicuity angle that explains what happens in the search experiment.

\section{Experiment 2}

The results of the first experiment show saccadic selection is dependent on the peripheral discrimination of the target. In the $l s f$ condition, search times are longer when the target is placed among similar flankers opposed to dissimilar flankers, an effect that reverses in the hsf condition. Even though we explain this result in terms of a contrast between similar and dissimilar flankers, one could argue that in both conditions clusters with $h s f$ flankers are simply fixated more frequently. We wanted to verify that the preference for clusters with similar flankers did not stem from a preference for clusters with a specific type of flankers.

Therefore, in this second experiment, a comparison is made between two conditions where the spatial frequency of target and flankers are equal. We now define the target in a feature space that should not influence its peripheral discriminability. Depending on the condition, the target is now either a green or a red vertical Gabor. All Gabors are at the high spatial frequency $\left(5.2 \mathrm{cycles}^{\circ} /{ }^{\circ}\right)$ of Experiment $1 \mathrm{~A}$, as we are interested in the generalizability of the preference for areas with stronger crowding.

\section{Methods}

Experimental procedures were similar to those of the first experiment. Below, we only discuss adaptations to the methods of Experiment 1A.
(B)

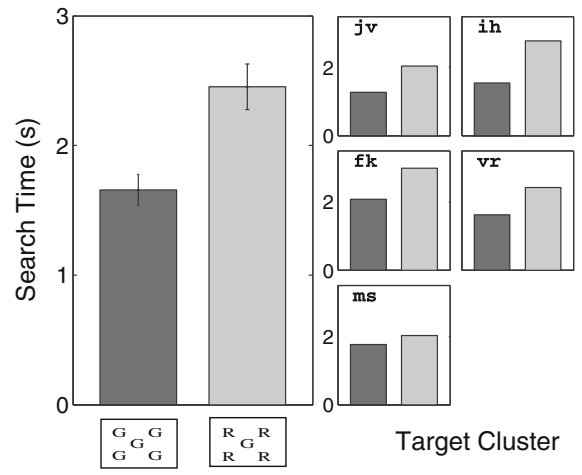

Light gray bars indicate search times for targets flanked by the opposite colored Gabors (dissimilar flankers). Letters represent cluster compositions, the $R$ 's indicate red Gabors, and the $G$ 's indicate green Gabors. Error bars represent standard errors of the mean

\section{Observers}

Five observers from the same pool as the previous experiment participated in the task. Two of those observers are also authors of this paper.

\section{Stimuli}

In the previous experiment, the target was distinguished by a difference in spatial frequency; here, the target was either a red or green Gabor (6.5 and $10.5 \mathrm{~cd} / \mathrm{m}^{2}$, respectively). In this experiment, all Gabors were of high spatial frequency $\left(5.2\right.$ cycles $\left.^{\circ}\right)$. Each condition included 150 stimulus displays. The target was a red vertical Gabor in the red condition, and the target was a green vertical Gabor in the green condition.

\section{Results}

Figure 6 displays the average search times. In this case, observers show the same pattern for both conditions. Search times are shorter when a target is flanked by Gabors of the same color than when the flankers have the opposite color $(t(5)=5.105 ; P<0.01$ and $t(5)=2.845 ; P<0.05$, for the red and green, respectively). Here, eye movements are attracted to clusters with strong crowding in both conditions.

In Fig. 7, we plot the total number of fixations on each type of cluster. Again the endpoints of the first three saccades are used in this analysis. The differences in these numbers clearly indicate a preference toward clusters where the flankers share the target color, rather than where the flankers have the opposite color. Clusters containing red flankers attract more saccades when a red target has to be found, and clusters containing green flankers attract more saccades when a green target has to be found. 
Fig. 7 Fixation frequency for the different cluster types. a Shows which clusters are fixated in the red target condition. b The fixations for the green target condition. Cluster compositions are represented by letters, $R$ 's indicate red Gabors, and $G$ 's indicate green Gabors. Error bars represent standard deviation
(A)
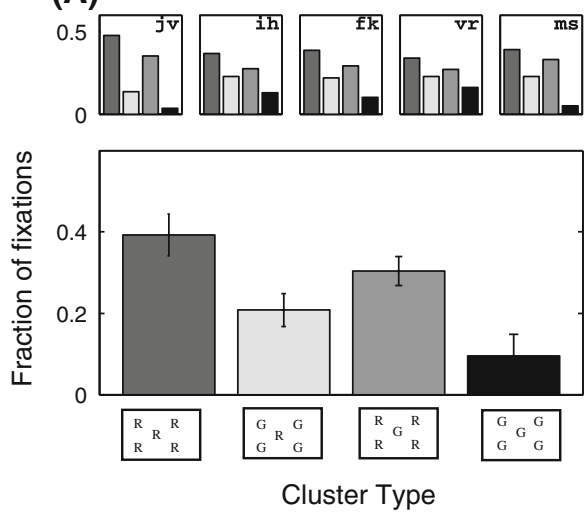

(B)
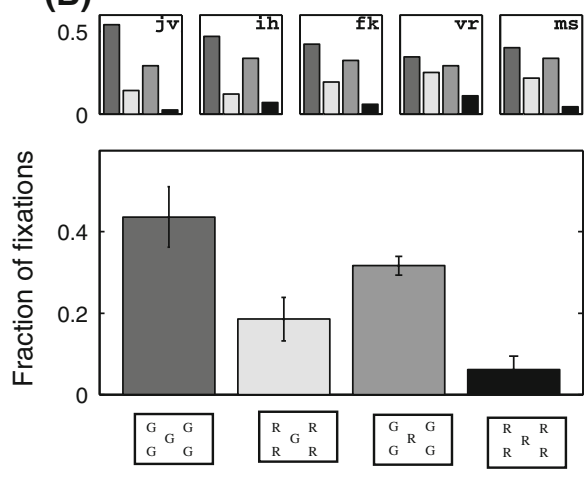

Cluster Type

\section{Discussion: Experiment 2}

In line with the findings from Experiment 1A, flankers have an effect on selection. In this experiment, targets were not peripherally discriminable. Here, we found search times to be shorter when a target is placed among flankers of the same color. As both targets are equally prone to crowding, the only difference that explains the results is a preference for clusters where flankers are similar to the target. The difference is more pronounced in the green condition than in the red condition. A likely reason for this anisotropy is that the red and green Gabors do not have the same onscreen luminance. However, even though red Gabors have a lower onscreen luminance than green Gabors, the general fixation patterns are similar.

The fixation patterns found in the second experiment show that selection is biased toward clusters with targetcolored flankers. Even though those clusters are fixated most frequently when they have a target-colored element at their center, when this is not the case, they are still fixated frequently. This gives rise to the question whether there is a continuous relation between the likelihood of selecting a cluster and the number of elements that hold the target property. Furthermore, as the results are consistent over targets defined by spatial frequency and color, we ask whether they also extend to a class of stimuli more frequently found in everyday life, such as letters. We investigated these two questions in the third and fourth experiment.

\section{Experiment 3}

A preference for clusters with many elements similar to the target can be seen in the fixation patterns from the previous experiment. However, the displays used only include a choice between clusters with strong crowding or very little. It is therefore not possible to conclude how sensitive the oculomotor system is if crowding was to vary more widely throughout the stimulus. The preference for clusters with strong crowding might be a very coarse one, only distinguishing between clusters with strong crowding and little crowding. However, it is also possible that saccadic selection is sensitive to a broad range of variations and that locations are compared on the basis of how strong the crowding is in a continuous relation. To test this, the composition of the clusters has been adapted. No longer are all flankers either similar or dissimilar, but their color is varied individually. The experiment was limited to just one condition with a red vertical target, as Experiment 2 demonstrated that a search for a red as well as a green target resulted in the same behavior.

Methods

\section{Observers}

Four observers of ages 25-28 participated in the third experiment. They were selected from the same pool as the previous experiments.

\section{Stimulus}

Six different cluster compositions can be distinguished, varying in the number of red (i.e., target colored) Gabors that a cluster contains (the complement of the Gabors in the cluster are green). The spatial frequency of these Gabors is the same as the high spatial frequency from Experiment 1 . A single display contains three clusters of each type, making for a total of 18 clusters in each display. The target, the only red vertical Gabor, is always presented at the center of a cluster. However, a red Gabor was not always presented at the center of a cluster not containing the target. There were 375 trials, balanced to be able to compare five subsets. These subsets were based on the number of red Gabors in the cluster containing the target. Each subset included all trials in which the target was found in a cluster with one, two, three, four, or five red Gabors, respectively. 
Fig. 8 Search times for the search for a red Gabor set out against the number of red Gabors in the target cluster (the complement is made up of green Gabors). Error bars represent standard errors of the mean

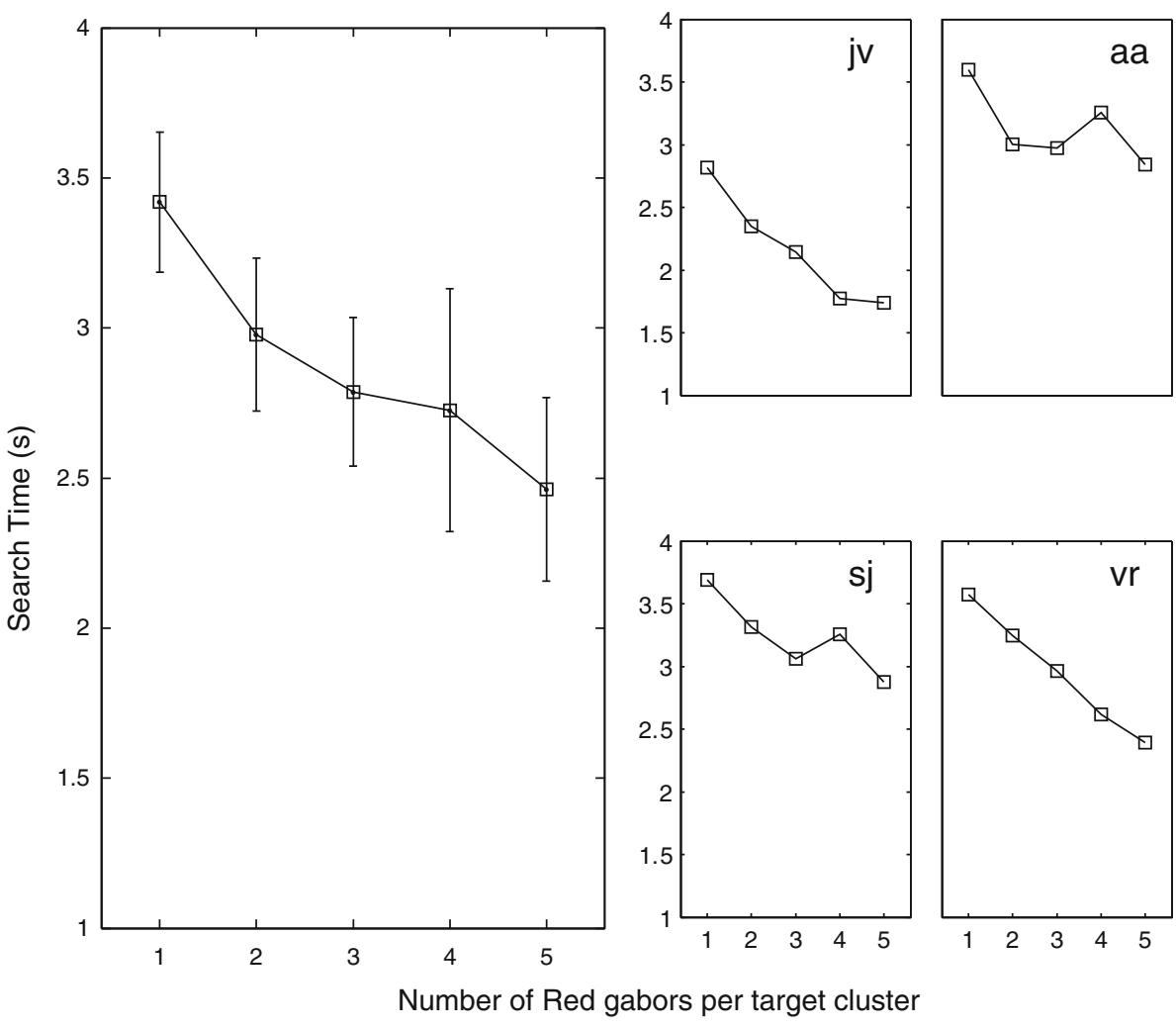

\section{Results}

Search times are set out against the number of red Gabors contained in a cluster and are found in Fig. 8. Search times decrease in a close to linear fashion (repeated measures ANOVA $F(4,16)=15.70, P<.001$, with a follow-up polynomial contrasts indicating a significant linear effect, $(F(1,5)=27.79, P<.05))$. In Fig. 9, we look at fixation data. As the target can now be present in a greater number of cluster types and search has become more extensive, we evaluate the first eight saccade endpoints. While search times were not always completely linear for each observer, in Fig. 9, we see the proportion of fixations for each cluster type does rise with each additional red Gabor presented in a cluster for all four observers (repeated measures ANOVA $F(5,20)=31.85, P<.001$, with a follow-up polynomial contrasts indicating a significant linear effect $(F(1,6)=$ 41.46, $P<.01))$.

\section{Discussion: Experiment 3}

The results again emphasize that the proportion of targetcolored Gabors over an area larger than that of just the target can attract saccades. Rather than a preference for salient structures, preference seems to be determined by the number of target-colored Gabors. Interestingly, even though the target cannot be discriminated peripherally, properties describing the area in which it lies are available for saccadic target selection.

Because in this experiment the probability of finding a target is equal for the different cluster types, there is no benefit of selecting areas with more elements similar to the target. However, this is only due to the artificial nature of the task, the target location is limited to the center of an isolated cluster, a restriction not common in everyday life. In daily life, selecting locations with many potential targets and strong crowding allows us to evaluate more potential targets upon fixation, which is probably an efficient strategy.

\section{Experiment 4}

The previous experiments show a bias in selection toward clusters with stronger crowding. This search experiment tests whether these findings can be generalized to a class of stimuli more common in everyday life. Here, the displays consisted of letters, frequently used in crowding experiments. We consider these stimuli more complex than Gabors, as they can be decomposed into parts of different orientations. These parts have different curvature, and finally, these parts in some cases intersect each other. 
Fig. 9 Fixation proportion while looking for the red vertical Gabor. The $y$-axis shows the relative number of fixations over all trials set out against the number of red Gabors per clusters. Each cluster holds five Gabors; the complement is made up out of green Gabors. Error bars represent standard deviations

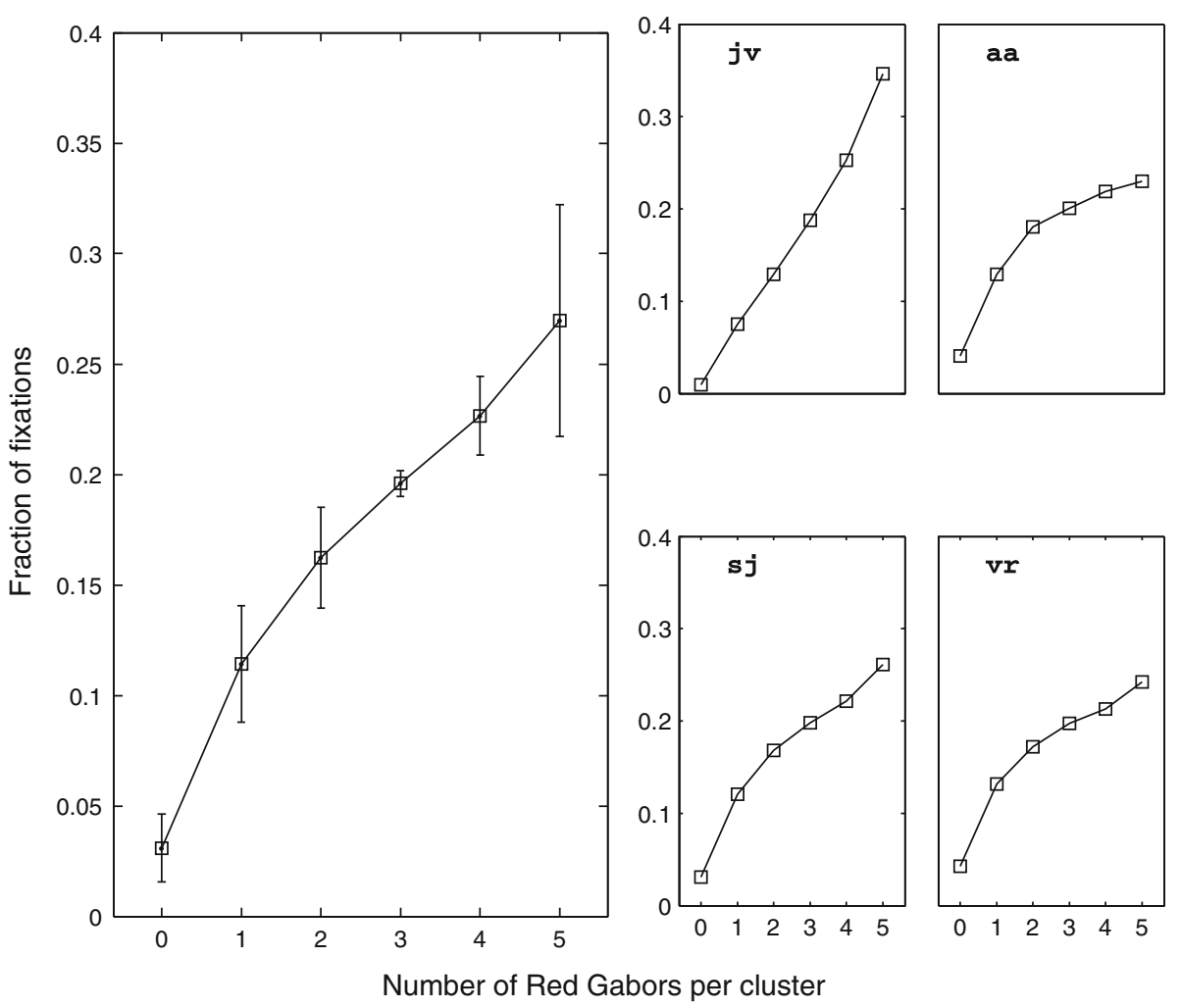

Methods

The general setup is again the same as that of experiments $1 \mathrm{~A}$ and 2. Only those methods that differ are discussed.

\section{Observers}

Six observers of ages 25-32 from the same pool participated in the task.

\section{Stimulus}

Each display contains a target $\mathrm{X}$ at the center of one of the 12 clusters. The Michelson contrast of all of the letters is equal. The clusters are composed in a similar fashion as in the first two experiments. A target $X\left(15 \mathrm{~cd} / \mathrm{m}^{2}\right)$ and distractors, 6 O's $\left(15 \mathrm{~cd} / \mathrm{m}^{2}\right)$ and $5 \mathrm{Y}$ 's $\left(15 \mathrm{~cd} / \mathrm{m}^{2}\right)$ are placed on an invisible hexagonal grid. Half of the letters are flanked by Y's, and the other half are flanked by O's. The choice for Y's and O's is based on their varying similarity to the $\mathrm{X}$. As the $\mathrm{X}$ and $\mathrm{Y}$ share more features than the $\mathrm{X}$ and $\mathrm{O}$, they can be used to place a target $\mathrm{X}$ either among similar or dissimilar surroundings, respectively.

\section{Results}

Figure 10 shows the search times are in line with the findings of the first two experiments. The displays where the $\mathrm{X}$ is

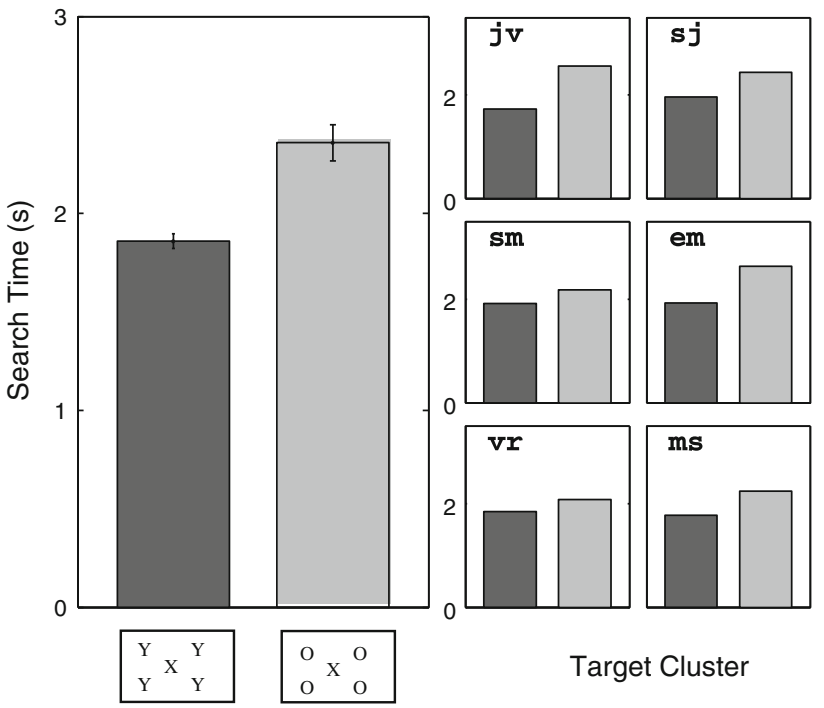

Fig. 10 Average search times. The dark gray bar indicates average search times for the subset where Y's accompany the target. The light gray bar indicates the average search times when $O$ 's accompany the target. Error bars represent standard errors of the mean

flanked by (the more similar) Y's result in shorter search times than those where (the more dissimilar) O's flank the X.

The proportion of fixations for each cluster can be found in Fig. 11. The analysis included the first three saccades of every trial. From this graph, it is clear that clusters where the flankers are Y's are selected more frequently than those 

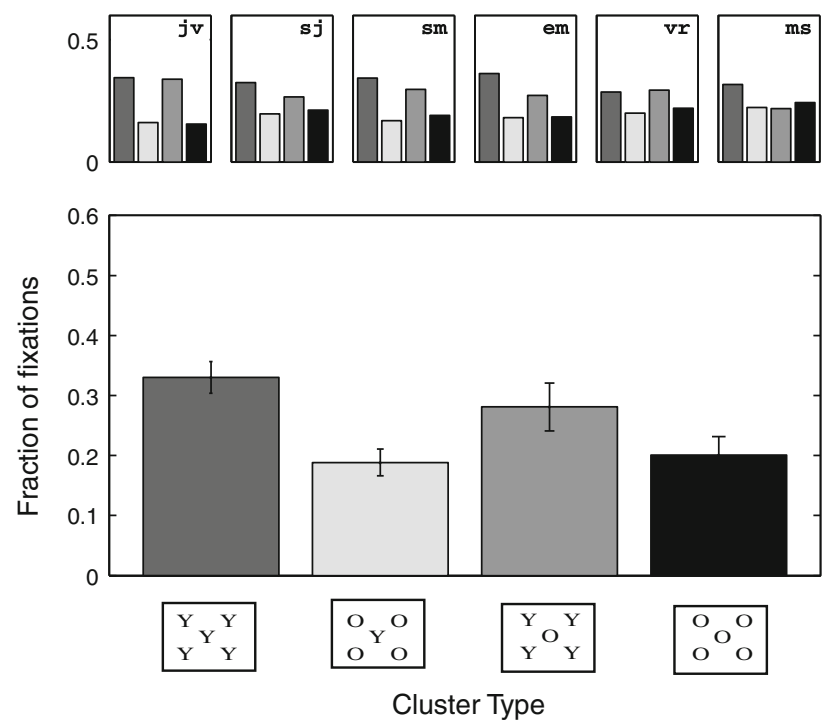

Fig. 11 Number of fixations for each cluster type. Cluster compositions are indicated by the letters that make up the cluster. Clusters that have a $Y$ at the center can also have the target $X$ at their center. Error bars represent standard deviations

where the flankers are O's. The bias toward the clusters with similar flankers is similar to those found in the previous experiments.

These results show that flankers affect saccadic selection in a similar fashion when the object of the search is more complex than a Gabor. As in Experiment 1A and 2, when crowding prevents the peripheral discrimination of the target, a bias toward areas with more of the target property is found.

\section{General discussion}

To investigate saccadic selection in relation to crowding, in four search experiments, we accompanied all individual search elements by flankers. To vary the strength of crowding on the search elements, these flankers could be either similar or dissimilar to their respective search element. In all experiments crowding influenced saccadic selection, however, not always in the same way. The oculomotor system preferred search elements in areas with little crowding for peripherally discriminable targets. However, interestingly, the oculomotor system preferred search elements with strong crowding, when looking for a peripherally indiscriminable target. We see this duality in Experiment $1 \mathrm{~A}$, where a low spatial frequency (lsf) target is found fastest when surrounded by flankers of a high spatial frequency ( $h s f$ ), while an $h s f$ target, peripherally indiscriminable, is found fastest among flankers of the same spatial frequency. The second experiment confirms the preference for areas with strong crowding when looking for a peripherally indiscriminable target. Here, looking for a red or green $h s f$ target, flankers of the target color attract the most eye movements. Vlaskamp and Hooge (2006) have shown that performance in visual search deteriorates with crowding. Interestingly, our findings show that crowding not just deteriorates peripheral target discrimination but also has an effect on the nature of potential targets in visual search. We see that when a target is peripherally indiscriminable, selection is biased toward areas with strong crowding. Even though adding flankers in a search task decreases overall search performance (Vlaskamp and Hooge 2006), surprisingly, the target is not necessarily found slowest in an area where crowding is the strongest. It appears similar flankers not only mask the signal of the target but can also attract eye movements as they raise the presence of target signal throughout the area.

The current experiments provide insight into the crowding phenomenon in general. There is still a lively debate on the mechanism behind crowding. Pelli et al. (2004), for instance, assume that crowding is due to a lack of featurebinding of individual elements (attributing features to corresponding elements correctly). Strasburger (2005) provides support for this theory, showing that observers often report a flanker instead of the target in letter identification tasks. Cavanagh (2007), on the other hand, advocates that averaging to the benefit of peripheral texture detection might be the underlying cause. In line with this thought, Saarela et al. (2009) show that when flankers group with other flankers, their masking strength decreases. As peripheral visual information can be used for saccadic selection, what is selected by eye movements cannot reveal the mechanism behind crowding, but can bring us more insight into what information is available in a crowded region (a region made up of a target and masking flankers). That is, finding that one type of cluster is preferred over another type also implies there is sufficient information on the visual aspects of these clusters to be able to distinguish between them. The results from Experiment 3 , showing that there is a continuous relation between the number of fixations on a cluster type and the number of target-colored Gabors, not just emphasize that eye movements are attracted by areas with more targetcolored elements. They also demonstrate that even though individual elements cannot be distinguished, information on the composition of the cluster is available as selection discriminates between clusters containing either more or less potential targets. This is interesting, as most suggestions on how crowding influences the percept are based on indirect measures. Saccadic targeting, however, reveals that enough information is present to be able to distinguish between different clusters. Even though peripheral target discrimination of an individual element fails, the clusters' contents can be evaluated. 
A preference for one type of cluster does not lead to any benefits in the present experiments. The target can be found just as likely in each type of cluster. However, in everyday life, items we try to locate are generally not limited to a specific position within an arrangement, and selecting areas with more elements holding target properties allows for evaluation of more potential targets upon foveation. We expect that the bias found is likely part of a search strategy, which includes two types of saccades that can be characterized as target directed and explorative. Target directed saccades occur when a target can be discriminated peripherally. These saccades target individual elements that could potentially be the target. Explorative saccades arise when a target is masked and saccades are directed toward areas with more potential targets and also stronger crowding. Together, these two types of saccades may provide for an efficient strategy to visual search in everyday life. When the target of a search stands out from its surroundings, it is selected directly. When the target does not sufficiently stand out from its surroundings, as a second best solution, saccades are directed toward areas with more of the target property present.

For instance, imagine you are looking for one of your books in your office, without any recollection of where you left it. This scene is likely to contain a bookcase, a location with lots of books and strong crowding. There might also be some books on your desk where there is less crowding, but also fewer books. To quickly find your book, an efficient strategy could be to make an eye movement to your bookcase, rather than one toward your desk.

Of course, to make the claim that this strategy is beneficial in everyday life, it is important that these results also hold for stimuli found in daily life. To a certain extent, our fourth experiment demonstrates that. The findings do not just hold for displays containing Gabors, but for letters as well.

Many studies evaluating the nature of potential targets have shown that features of peripheral elements play an important role in saccadic selection (e.g. Findlay 1997; Hooge and Erkelens 1999; Motter and Belky 1998; McSorley and Findlay 2003). These studies evaluate saccadic selection on the basis of single elements on a sparse grid and show target similarity to be an important factor in guiding eye movements. In studies using more complex scenes, area composition has been taken into account (e.g., Neider and Zelinsky 2006; Torralba et al. 2006; Shen and Pare 2006). However, emphasis lies on saccadic selection guided by salience, rather than by target similarity. The current study evaluates the nature of potential targets on the basis of the combination of elements near each other in the periphery, in a complex search task. When a target can be discriminated peripherally, target similarity is highest when crowding is weak. However, when a target cannot be discriminated peripherally, due to crowding, target similarity seems to be estimated over a larger area. Therefore, the role of flankers similar to the target in visual search is two-fold. They impair peripheral target discrimination, and-interestingly - they also attract a greater number of eye movements, as more of the target property is present at the location of the target.

Open Access This article is distributed under the terms of the Creative Commons Attribution Noncommercial License which permits any noncommercial use, distribution, and reproduction in any medium, provided the original author(s) and source are credited.

\section{References}

Avraham T, Yeshurun Y, Lindenbaum M (2008) Predicting visual search performance by quantifying stimuli similarities. J Vis 8:1-22

Bacon WJ, Egeth HE (1997) Goal-directed guidance of attention: evidence from conjunctive visual search. J Exp Psychol Hum Percept Perform 23:948-961

Bouma H (1970) Interaction effects in parafoveal letter recognition. Nature 226:177-178

Burr DC, Wijesundra SA (1991) Orientation discrimination depends on spatial frequency. Vis Res 31:1449-1452

Butler BE, Currie A (1986) On the nature of perceptual limits in vision: a new look at lateral masking. Special Issue: Visual Selective Attention. Psychol Res 48:201-209

Cavanagh P (2007) Seeing the forest but not the trees. Nat Neurosci 4:673-674

Duncan J, Humphreys GW (1989) Visual search and stimulus similarity. Psychol Rev 96:433-458

Findlay JM (1997) Saccade target selection during visual search. Vis Res 37:617-631

Findlay JM, Brown V (2006) Eye scanning of multi-element displays: II. Saccade planning. Vis Res 46:216-227

Fredericksen RE, Bex PJ, Verstraten FA (1997) How big is a Gabor patch, and why should we care? J Opt Soc Am A Opt Image Sci Vis $14: 1-12$

Hooge ITC, Erkelens CJ (1999) Peripheral vision and oculomotor control during visual search. Vis Res 39:1567-1575

Huckauf A, Heller D, Nazir TA (1999) Lateral masking: limitations of the feature interaction account. Percept Psychophys 61:177-189

Jacobs AM (1986) Eye-movement control in visual search: how direct is visual span control? Percept Psychophys 39:47-58

Kooi FL, Toet A, Tripathy SP, Levi DM (1994) The effect of similarity and duration on spatial interaction in peripheral vision. Spat Vis 8:255-279

Levi DM (2008) Crowding — an essential bottleneck for object recognition: a mini-review. Vis Res 48:635-654

Luria SM, Strauss MS (1975) Eye movements during search for coded and uncoded targets. Percept Psychophys 17:303-308

McSorley E, Findlay JM (2003) Saccade target selection in visual search. Accuracy improves when more distractors are present. $\mathrm{J}$ Vis 3:877-892

Motter BC, Belky EJ (1998) The guidance of eye movements during active visual search. Vis Res 38:1805-1815

Nazir TA (1992) Effects of lateral masking and spatial precueing on gapresolution in central and peripheral vision. Vis Res 32:771-777

Neider MB, Zelinsky GJ (2006) Searching for camouflaged targets: effects of target-background similarity on visual search. Vis Res 46:2217-2235

Pelli DG, Palomares M, Majaj NJ (2004) Crowding is unlike ordinary masking: distinguishing feature integration from detection. J Vis 4:1136-1169 
Polat U, Sagi D (1993) Lateral interactions between spatial channels: suppression and facilitation revealed by lateral masking experiments. Vis Res 33:993-997

Saarela TP, Sayim B, Westheimer G, Herzog MH (2009) Global stimulus configuration modulates crowding. J Vis 9:1-11

Shen K, Pare M (2006) Guidance of eye movements during visual conjunction search: local and global contextual effects on target discriminability. J Neurophys 95:2845-2855

Strasburger H (2005) Unfocussed spatial attention underlies the crowding effect in indirect form vision. J Vis 5:1024-1037

Toet A, Levi DM (1992) The two-dimensional shape of spatial interaction zones in the parafovea. Vis Res 32:1349-1357

Torralba A, Oliva A, Castelhano MS, Henderson JM (2006) Contextual guidance of eye movements and attention in real-world scenes: the role of global features on object search. Psychol Rev 113:766-786
Treisman AM, Gelade G (1980) A feature-integration theory of attention. Cogn Psychol 12:97-136

Van Der Steen J, Bruno P (1995) Unequal amplitude saccades produced by aniseikonic patterns: effects of viewing distance. Vis Res 35:3459-3471

Vlaskamp BNS, Hooge ITC (2006) Crowding degrades saccadic search performance. Vis Res 46:417-425

Wertheim AH, Hooge ITC, Krikke K, Johnson A (2006) How important is lateral masking in visual search? Exp Brain Res 170:387-402

Wolfe JM (2007) Guided search 4.0: current progress with a model of visual search. In: Gray W (ed) Integrated models of cognitive systems. Oxford, New York, pp 99-119

Yarbus AL (1967) Eye movements and vision. Plenum Press, New York

Zelinsky GJ (1996) Using eye saccades to assess the selectivity of search movements. Vis Res 36:2177-2187 